identical refractions; $(2)$ that the refractions in the case of identical twins are alike at least twice as often as they are in the case of brothers and sisters of the same family born at different times; (3) the occurrence of strabismus is higher in twins than in sibs of different ages--it occurs sometimes in one member of the pair only, but far more commonly in both members, and then is of the same nature and direction in each.

\title{
REFERENCE
}

Wilson, J. A.-Twins with eye defects. Ametropia and Strabismus. Brit. Jl. of Ophthal., Vol. XVI, p. 421, July, 1932.

\section{SOME PRACTICAL POINTS IN THE TREATMENT OF SIMPLE DETACHMENT OF THE RETINA}

\author{
B Y \\ HaROLD RIDLEY \\ SENIOR RESIDENT OFFICER, MOORFIELDS EYE HOSPITAL
}

OF late, with improvements in technique, operations for detachment of the retina have become much more frequent. Though at the moment diathermy operations are in almost universal favour, there is considerable variation in method and application, and a few notes based on first-hand experience, either as operator or assistant, of a large number of cases recently dealt with at Moorfields may be of interest.

The problem, like any other in surgery, consists of two partsdiagnosis and treatment. From the surgical aspect a simple retinal detachment should be regarded as a secondary lesion following a retinal tear, and on this latter our attention must be concentrated. Diagnosis consists of localization of the hole.

Localization of the hole may be simple or very difficult. In many cases a very shrewd idea can be obtained even without the use of the ophthalmoscope. A patient seen wearing a high myopic correction is most likely to have a large tear in the upper half of the retina some distance from the periphery; an emmetrope or hypermetrope a disinsertion in the lower temporal quadrant. The patient's history, if reliable, is of great importance ; for instance, flashes of light in the lower nasal field followed by a shadow beginning in the same situation is almost pathognomonic of a hole in the upper temporal quadrant. Unfortunately, a shadow beginning in the upper half of the field is not such a reliable guide to the situation of the hole, for subretinal fluid leaking through a hole above tends to sink under the action of gravity 
and may float-off the lower half of the retina before the upper. Even then the history may tell whether the hole is temporal oro nasal, a valuable point if its exact localization be difficult or eveno impossible. In cases of direct trauma the site of impact is a useful $\frac{\bar{\omega}}{\overrightarrow{5}}$ guide, e.g., a patient who in stooping struck her eye on the handle of a saucepan was found to have a tear in the lower temporale quadrant where the injury occurred. Finally patients giving a $\vec{\circ}$ long history of loss of the upper half of the field are almost certain to have a disinsertion and not a true tear, but a stationary or $\vec{\omega}$ very slowly progressive detachment in the upper part of the fieldo is highly probably a cystic detachment. These retinal cysts are? for practical purposes to be considered as simple detachments forco they take the same course, though generally in longer time, and call for similar treatment. They may be diagnosed on the following $\vec{a}$ characteristics. They generally occur in the temporal quadrants,, usually the upper, in persons of middle age; their onset is suddenor at least noticed suddenly, after which progression, if any, is ${ }_{\mathbb{D}}^{\top}$ very slow. On examination the vitreous is usually absolutely을 clear and the detachment looks particularly thin and transparent. The edge, if visible, is sharply defined and rather crenated. The detached retina looks very thin, suggesting that the separatione occurs somewhere between the layers of the rods and cones and the ganglion cells, and not between the rods and cones and pigme ito layer, as in other cases. Permanent loss of field in the detachmentt area, even after replacement is evidence in favour of this. Finally, though the whole surface of the detachment may be perfectly examined no hole is found, but even before this final conclusive test, if such be possible in the particular case, the diagnosis should요물 have been made. These cysts constitute something like 3 per cent. of the cases.

The examination should consist first in mapping out the totalo extent of the detachment by the ophthalmoscope and perimeter, though it must be remembered that detached retina is sometimes? sensitive. The field is also useful for comparison before and aftero operation. The suspected quadrant should then be searched in detail for the hole, beginning at the periphery where most occur.o At this stage two tactors are of the greatest value, the maximal dilatation of the pupil, obtained if necessary by the use of mydricaine, and the co-operation of the patient who must rotate the eye to the maximum in the direction required for examination of the particular area of peripheral retina being searched. An ophthalmoscope with a focusing beam of light permits much morew detailed examination of any particular area of the retina and is also of great value in cases where the view is obstructed by opacities? in the lens or vitreous. The finding of one hole by no meanss rules out the existence of others which may perhaps be secondaryo 
to the detachment. As many as five, some probably secondary, are not infrequent in highly myopic cases. In cases of detachment following a spontaneous vitreous haemorrhage the hole may be seen involving an area crossed by a large vessel.

The hole when sighted should be orientated and this is conveniently done in "o'clocks" as though the patient's eye looked at from the front were the face of a watch. This part is usually fairly simple, the more difficult being to decide how far back the hole is situated. The old method of measuring the number of disc diameters between the hole and periphery, multiplying by $\mathbf{1 . 5}$ (D. D. $=1.5 \mathrm{~mm}$.) and adding 8 or $9 \mathrm{~mm}$. for the distance between the limbus and retinal periphery usually under-rated the distance considerably. If this method must be employed it is better to allow $2 \mathrm{~mm}$. for each D. D. and $10 \mathrm{~mm}$. for the distance between the limbus and retinal periphery. The method usually employed at Moorfields is based on practical experience. In an emmetropic eye a diathermy application $12 \mathrm{~mm}$. from the limbus will be just visible in its entirety at the periphery and one $22 \mathrm{~mm}$. back will be as far back as a hole is ever seen, almost at the macula. Between these extremes judgment is exercised, bearing in mind that most errors are due to under rather than over estimation-a rather more generous estimation should be made in highly myopic eyes. It is difficult to determine exactly the real situation of a hole placed on a ballooned part of retina and here again judgment must be exercised.

Finally, the condition of the macula should be recorded. If normal, $6 / 6$ vision may naturally be expected immediately after the operation. If moderately involved, a reddish area surrounded bv white oedema, vision may return to $6 / 6$ in the course of months. If a "hole at the macula" be seen good central vision is permanently lost. The condition of the macula determines the urgency of operation, a detachment spreading almost to the macula clearly necessitates early operation, or at least absolute rest in bed, whereas an interval of a week or two makes little difference if the macula be entirely off. Occasionally one sees an early case in which a large balloon detachment is present above overhanging, but not otherwise affecting the macula. In the writer's opinion such cases are as much surgical emergencies as an acute appendix and even delay in bed is unjustifiable.

In the operation itself there are two things essential, the causing of a plastic choroiditis in the right region to seal the hole, and draining the fluid. Of the two the latter, though it tends to be somewhat ignored, is if anything the more important. If the retina fails to fall against the prepared choroid the effect of the diathermy is wasted, and moreover, damage has been done to the sclera which makes further operation more difficult. 
In cases where no hole is found reliance must be placed on the $\overrightarrow{\vec{F}}$ patient's history (vide supra) as to where flashes of light and the shadow first appeared. This is of particular importance in aphakic cases where it may be impossible to examine the periphery, owing $\frac{\bar{\omega}}{\sigma}$ to the presence of capsule and loss of the prismatic effect of the $\mathbb{0}$ lens. In cases in which numerous scattered holes exist it is suggested that diathermy be performed over the upper half of the retina and the fluid drained from below. With the patient propped up in bed the upper half may become attached and the $\vec{\omega}$ residual detachment below can be treated later by diathermy.

The general technique of the operation is well-known, but comment on various points may be of interest. Temporary division $\overrightarrow{0}$ of a muscle is called for in the majority of cases, even though ? the hole may not lie directly under a muscle belly. A far better $\vec{a}$ exposure is obtained making an extensive operation feasible. Heterophoria following this is of great rarity and seems to disappear in a short while. Traction by means of a strabismus T hook is a painful and much less effective method.

A stitch passed through the sclera is useful for marking the meridian and for traction. The further from the limbus it is placed the greater its leverage.

If it be necessary to apply diathermy far behind the equatơ the speculum may be removed and the lid and conjunctiva hef back by a Desmarres retractor. This gives an extra 2 to $3 \mathrm{~mm}$. exposure and is probably better than instruments which lever

forward the entire globe. application of the electrode used in Larsson's method. Nothing 9 but the tip of the electrode should touch the patient, current $\vec{F}$ too often leaks away through conjunctiva touching the shaft. The electrode should be pressed firmly against the sclera before the current is turned on and the area should be free from blood, but moistened by distilled water through which the current does not 3 leak which prevents charring of the sclera. If the sclera 8 be charred, little reaction is caused in the choroid.

Applications some distance from the hole join the retina and 9 choroid over a broad area and diminish the risk of secondary $>$ tears. The slight extra loss of peripheral field is usually negligible.

The dosage is important. Recently there has been a tendency $N$ to increase, with favourable results. Seventy milliampères of N current for seven or eight seconds appear to be most effective. If $O$ an overdose be given there is excessive exudation from the choroid by which the retina may be pushed forward temporarily. Though $\Phi$ this appears to settle there is an increased risk of vitreous? 
haemorrhage. Retinal haemorrhages are frequently seen in such casès.

Far back the vortex veins may be encountered. These should be avoided. They bleed freely and cases of massive thrombosis have been reported.

Trephining the sclera has generally replaced the cautery for drainage. A few brisk but light twists of the trephine are better than slower and more deliberate. A clean disc of sclera is removed and the dark choroid bulges slightly outwards. A straight puncture through the choroid with the punctum dilator is more likely to give continuous drainage than an oblique which may form a valvular opening. The drainage must take place from an area of deep detachment which must be kept dependent after the operation. For this reason one of the lower quadrants is preferable to an upper, the patient being more comfortable when propped up than when lying flat. The trephine should be placed in a white area of sclera free from vessels and not near a recently divided tendon where reaction may rapidly cause closure. In cases where much sub-retinal fluid is present, or where the sclera has not been cleanly trephined, allowing the choroid to project through the hole, a second scleral trephine may be performed before the choroid is punctured in either place. An alternative method is to drain the fluid by three or four Safar punctures.

At the end of the operation a little gentle massage, preferably under observation through an ophthalmoscope, is probably advantageous. With large holes, especially disinsertions, there is, however, a definite risk of pushing vitreous between choroid and retina if massage be excessive.

Of complications the worst is sepsis. Panophthalmitis has been seen by the writer only once, in a case in which abnormal technique was tried. Slight degrees, perhaps as much traumatic as bacterial, in the sub-conjunctival tissues are common. Chemosis must be regarded definitely as a bad sign for exudation of fluid externally is nearly always accompanied by fluid beneath the retina. Similarly the tension of the globe if anything but soft for the first few days denotes deficient drainage. Another serious complication is vitreous haemorrhage, which may be seen on the day following the operation, in which case its origin is probably from the choroid which may be wounded by the trephine, or about the third or fourth day, in which case it is usually associated with excessive diathermy and consequent retinal haemorrhage. In this connection it may be stated that a woman was discharged three weeks after operation cured but with a retinal haemorrhage. She returned a week later with a vitreous haemorrhage.

The direct causes of failure are two-fold-deficient drainage or lack of diathermy to close the hole. Of the two the latter is the 
rarer and usually easier to cure, for with the retina in place it is simple to apply diathermy under direct observation if necessary. Inefficient drainage may mean that the whole or part of the detachment remains. In some cases vitreous between choroid and retina prevents apposition of the layers. This is most common in myopes with enormous peripheral tears simulating disinsertions in which the torn edge is turned over like the page of a book.

The successful case shows in the first few days white exudates superficial to the retina. On the top of these a small haemorrhage is quite frequently seen. At the end of a fortnight retinal pigment disturbance appears, and this is a sure sign that the retina has been in contact with the inflamed choroid.

Most cases worth operating on once are worth a second attempt iv if the hole be unclosed. How soon after the first the second should be performed is a matter of opinion. Residual detachment in the lower half where the hole is sealed may be left, for the fluid absorbs spontaneously in time in many cases while the patient leads a normal life. Cases with a small residual detachment where closure of the hole is uncertain and the macula in danger should be proceeded with two or three weeks after the first operation before the patient is allowed out of bed. If the macula is no $\vec{\oplus}$ endangered it may be preferable to allow a longer interval, fo some of these subside spontaneously. A second operation soow. after the first is rendered difficult by adhesions between scler and conjunctiva, which not only bleed freely, but make application of the electrode to the sclera deficient, current leaking along adhesions, moreover, the trephine hole may be opened and the eye become soft. In this type of case the method of Safar, in which the electrode perforates the sclera is of value, as leakage along the surface is much less. The writer has only a small experience of this method which in ordinary cases appears to have no advantages and is unpleasant owing to leakage of sub-retinal fluid and softness of the globe during performance of the operation. If a very large residual detachment be present there is no cause for hurry and an interval of some weeks will make the second operation easier.

Certain cases are not worth further effort. Among these are those with much opacity in the vitreous, which is always rather unfavourable, and cases in which a large secondary disinsertion develops.

As a rule detachments of more than a year's standing are $N$ unlikely to be cured, though a successful result after four and a N half years has been seen, though in this case the macula did not $g$ regain its function.

I am indebted to the Staff of Moorfields for the material on which this paper is based. 